\title{
Observations of Interstellar Reddening in Cygnus and Perseus ${ }^{1}$
}

\author{
K. NANDY \\ Royal Observatory \\ Edinburgh, Scotland
}

THE OBJECT OF THE EDINBURGH SPECTROPHOTOMETRIC SURVEY is $1_{\text {to }}$ determine the interstellar extinction law as precisely as possible and to search for intrinsic variations in it. The observations have been extended to early type stars up to a limiting magnitude of $11 \mathrm{~m}^{\mathrm{m}} 0 \mathrm{in}$ regions extending from $\ell^{\mathrm{II}}=50^{\circ}$ to $\ell^{\mathrm{II}}=200^{\circ}$. The 16/24/60-inch Schmidt telescope of the Royal Observatory has been used in conjunction with an objective prism and grating. Spectra extend from $\lambda=3300 \AA$ to $\lambda=9000 \AA$ with a dispersion of $1000 \AA / \mathrm{mm}$ at $\mathrm{H} \gamma$. Since few stars fainter than $9^{\mathrm{m}}$ have been measured in earlier investigations, the present survey extends considerably the amount of information available.

In determining the extinction from a comparison of pairs of stars, one reddened and the other unreddened, one is faced by the problem of having to find two intrinsically similar stars in the same galactic region. Uncertainties in the assigned spectral types of stars give rise to systematic errors in the extinction curve. Such errors can be reduced by increasing the number of pairs of stars observed, and the study of interstellar extinction by earlier authors has been affected by the relatively small number of stars observed. The present paper describes the results for two regions in the direction of Cygnus $\left(\ell^{\mathrm{II}}=80^{\circ}\right)$ and Perseus $\left(\ell^{\mathrm{II}}=140^{\circ}\right)$.

\section{METHODS OF OBSERVATION AND MEASUREMENT}

The Edinburgh Schmidt telescope has a field diameter of about $4^{\circ}$. The performance of the telescope is described in reference 1 . The objective prism of angle 1:8, made of LF5 glass, has a high ultraviolet transmission. The spectra were broadened to $0.3 \mathrm{~mm}$ by varying the sidereal rate.

${ }^{1}$ The contents of this paper were published previously in the Pub. Roy. Obs. Edinburgh, vol. 3,1964 , p. 142 , and vol. 5,1965 , p. 13. 
In order to reduce overlapping of spectra, different emulsion-filter combinations were used to restrict the length of spectra and several have been found suitable. The plates were calibrated from the known magnitude differences between center and first-order side images of prism-crossed-by-grating spectra. For wavelength identification, hydrogen lines as well as features such as the sharp cutoff wavelength of the emulsions provided suitable reference points.

The tracings were taken with a Joyce Loebl microdensitometer which records density $\left(D=\log _{10} 1 / T\right)$. Absolute density values were converted to "Baker densities" by using the equation

$$
\Delta=\log _{10} \frac{1-T}{T}
$$

where this modified function of the transparency $T$ is approximately linearly related to magnitude over a wide range of $T$. (See ref. 2.)

The monochromatic magnitude difference between a reddened and a comparison star of the same spectral type and luminosity class consists of two parts: (a) an intrinsic magnitude difference; and (b) a difference in the effect of interstellar extinction on these two stars.

If the reddened and the comparison stars are situated in the same galactic region, the extinction law and the energy distribution in the spectrum of the same type of star may be assumed to be the same. Monochromatic magnitude differences as functions of wavelength for any pair of two such stars should therefore represent effects of interstellar extinction.

The main sources of error are: (a) uncertainty in spectral classification; (b) difficulties in determining wavelengths at which observations are made; (c) graininess of plates; and (d) overlapping of spectra. Results of reference 3 show that the errors of the observed extinction curve arise primarily from uncertainties in spectral classification. An error of \pm 1 subclass in spectral type may result in an error of \pm 0.15 in the slope of the reddening curve in the ultraviolet relative to blue. Accuracy of spectral classification is therefore highly important; measuring the largest possible number of pairs of stars is useful.

\section{EXTINCTION LAWS FOR CYGNUS AND PERSEUS}

\section{Observations}

A region of 30 square degrees in Cygnus (centered on $20^{\mathrm{h}} 15^{\mathrm{m}}, 38^{\circ}$ ) and 40 square degrees in Perseus (centered on $2^{\mathrm{h}} 12^{\mathrm{m}}, 57^{\circ} 30^{\prime}$ ) has been investigated for which spectral types on the MK system exist.

As reported in references 3 and 4, 28 pairs of stars in Cygnus and 41 pairs of stars in Perseus have been measured. The extinction curves have been normalized so that the slope of the line fitted at $\lambda^{-1}=1.34 \mu^{-1}$ 
and $\lambda^{-1}=2.28 \mu^{-1}$ is unity, and the zero point is chosen such that the extinction $\Delta m_{\lambda}=1$ at $\lambda^{-1}=2.22 \mu^{-1}$. Magnitude differences for a number of pairs of stars have been compared with photoelectric measures and the agreement is good. (See ref. 3.)

\section{Extinction curves from $O$ and $B$ stars}

Results of reference 5 have suggested that a higher ratio of $(U-B)$ color excesses to $(B-V)$ color excesses for $O$ stars in Cygnus than found elsewhere is due to intrinsic ultraviolet deficiency in these stars. In this case the observed extinction effect for Cygnus stars should be a function of spectral type. In order to test this theory, the mean extinction curve derived from $O$ stars has been compared with that derived from $B$ stars. The difference is found to be within an observational error of \pm 5 percent both in Cygnus and in Perseus and has, therefore, no significance.

\section{Intrinsic rms dispersion}

Comparing the observed $\mathrm{rms}$ dispersion with predicted standard errors of observation reveals that the intrinsic dispersion in the extinction curves in either direction is less than 3 percent.

\section{Dependence on distance}

In each region of Cygnus and Perseus, all the stars which have been studied by present and earlier authors have been divided into two groups of approximately equal number:

\begin{tabular}{cccc} 
& & \multicolumn{3}{c}{ Mean $m-M$} \\
Group & $m-M$ & Cygnus & Perseus \\
I & $<12.0$ & 10.4 & 11.2 \\
II & $\geqslant 12.0$ & 12.0 & 12.4
\end{tabular}

where $m$ is the apparent magnitude and $M$ is the absolute magnitude. For each group magnitude differences have been calculated for pairs of highly and moderately reddened stars belonging to the same group. A third group of extinction curves has been obtained by comparing moderately reddened stars with nearby slightly reddened stars.

Neither in Cygnus nor in Perseus do the mean extinction curves derived for each of three different groups differ from each other by more than 4 percent. It is therefore concluded that in the directions of Cygnus and Perseus the extinction law is independent of distance out to at least 2.5 kiloparsecs.

\section{Weighted mean extinetion curves}

Since there are no significant differences in the extinction curves derived from different groups of stars, weighted mean extinction curves have been derived for the two regions, Cygnus and Perseus. The weight is equal to the difference in the color excesses of the pair of the stars compared. 
For the Cygnus region, all available observational data have been combined to give a weighted mean extinction curve with a standard error of $\pm 0.008^{\mathrm{m}}$. Spectrophotometric data of other observers for stars in the Perseus region are fewer than those for Cygnus stars. The mean extinction curve has therefore been obtained from the 41 pairs of stars studied by the author with a standard error of $\pm 0.01^{\mathrm{m}}$.

A comparison between the results for Cygnus and Perseus is shown in figure 1. Least-square fitting of the observed points of figure 1 by orthogonal polynomials shows that the best fitting for each of two curves is either two straight lines intersecting at $4300 \AA$ or polynomials of degree higher than three to give the variance $\sigma^{2}$ of the order of the standard error of the curve.

It is of interest that the curve for Cygnus stars in reference 6 appears to consist of two linear parts between $0.8 \mu^{-1}$ and $3.0 \mu^{-1}$.

The ratio of the slope of the ultraviolet part of the curve to that of the blue-visible part is 0.64 for the Cygnus curve, in agreement with the result of reference 6 , and 0.46 for the Perseus curve.

\section{Observations and theory}

The 30-percent difference in the slopes of the ultraviolet part of the reddening curves relative to the blue part appears to be in agreement

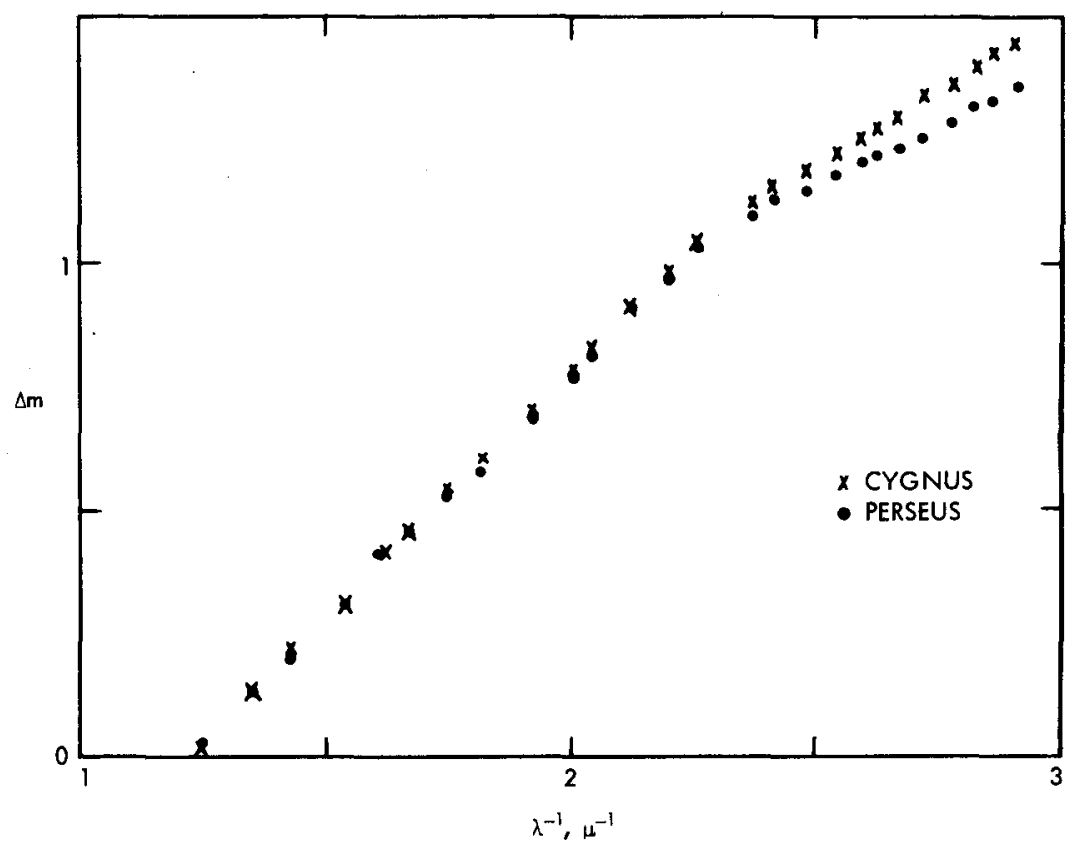

Figure 1. - Weighted mean extinction curve in directions of Cygnus and Perseus. 
with the prediction of reference 7 . A further requirement for the validity of the theory of reference 7 is that the ratio of the slope of the ultraviolet part to that of the blue-visible part should be dependent on the angle of viewing the spiral arm, or in other words, on the alinement properties of the particles and on the polarizability. (See ref. 8.) However, the dependence on galactic longitude has not been confirmed by the work reported in references 9 or 10.

The extinction of the Pleiades star HD 23512 offers a further test for the verification of the prediction of reference 8 . This star is reddened by an amount of 0.35 ; polarizability is the same as the average value in Perseus and about four times that in Cygnus.

This Pleiades star, which has been studied at Edinburgh, is also discussed in reference 11. The slope of the ultraviolet part of the Pleiades extinction curve (fig. 2) relative to the blue-visible part is essentially the same as that found for the Cygnus curve; the small difference in the infrared may not be significant. The variation in the extinction law cannot be explained, therefore, by differences in alinement of the particles. An alternative possibility is that differences in composition of the particles may be responsible for the variation of the extinction law.

Figures 3 and 4 present comparisons of the observed extinction law

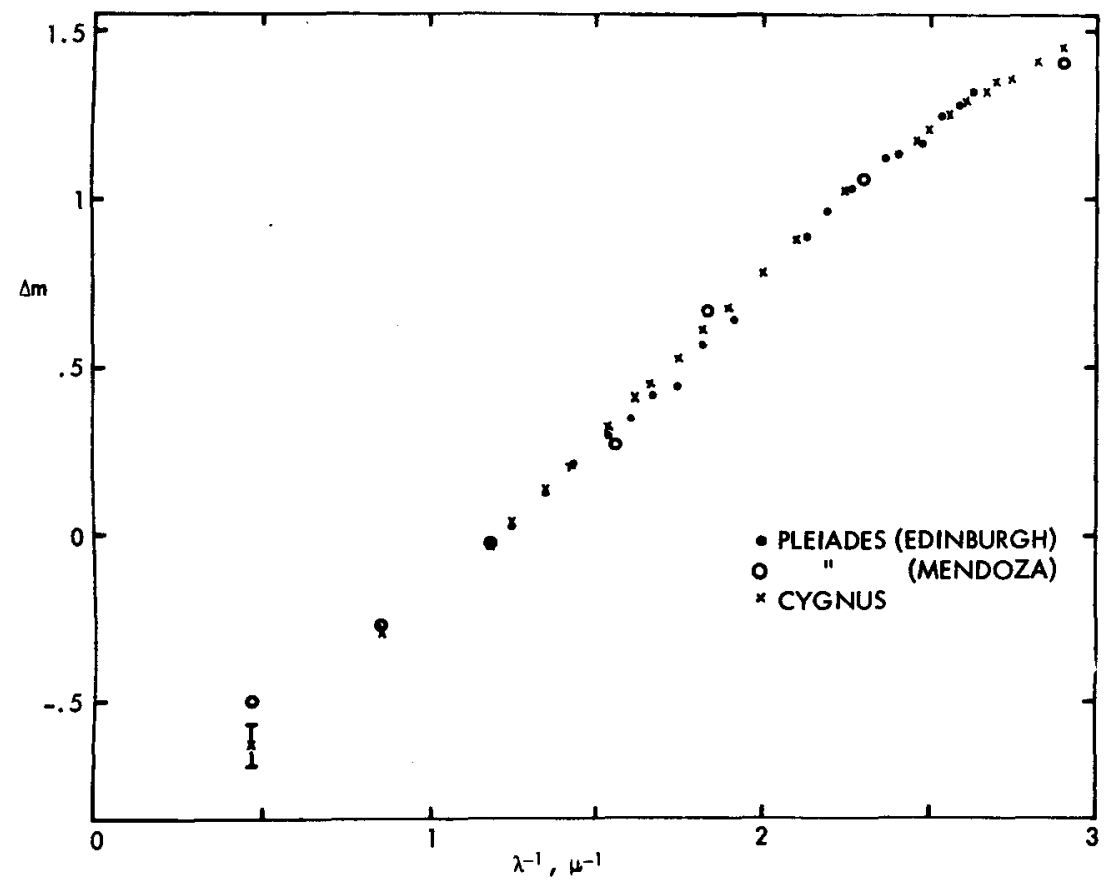

FIGURE 2.-Pleiades extinction curve. Vertical line denotes uncertainty of data point. 


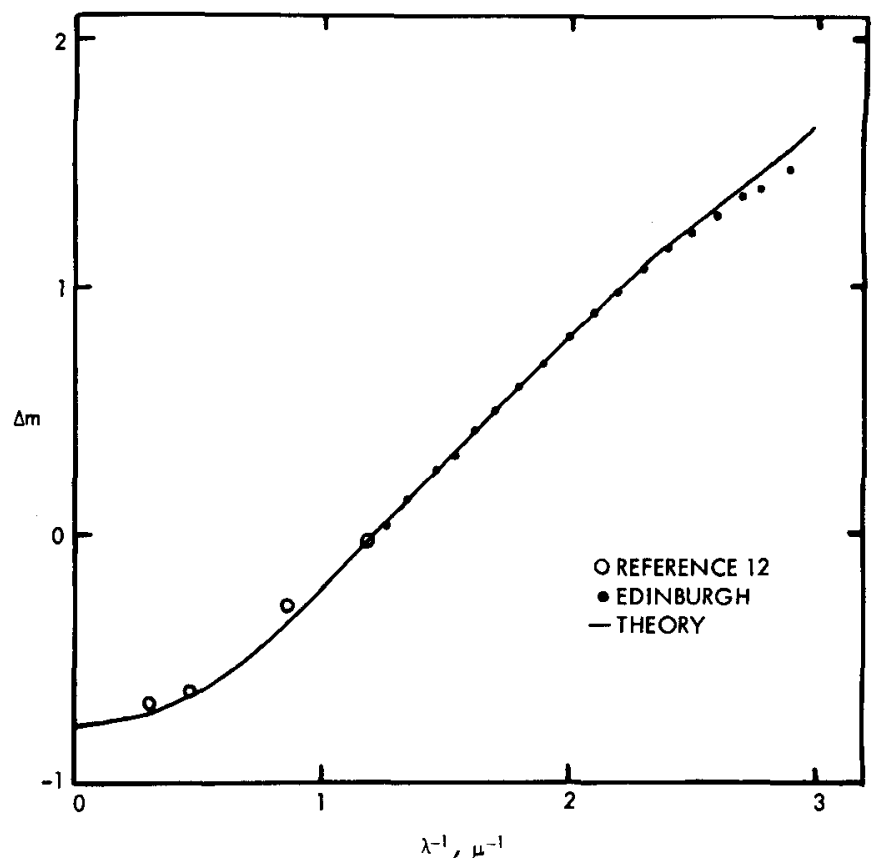

Figure 3. - Comparison of observed extinction law in Cygnus with theoretical extinction curve.

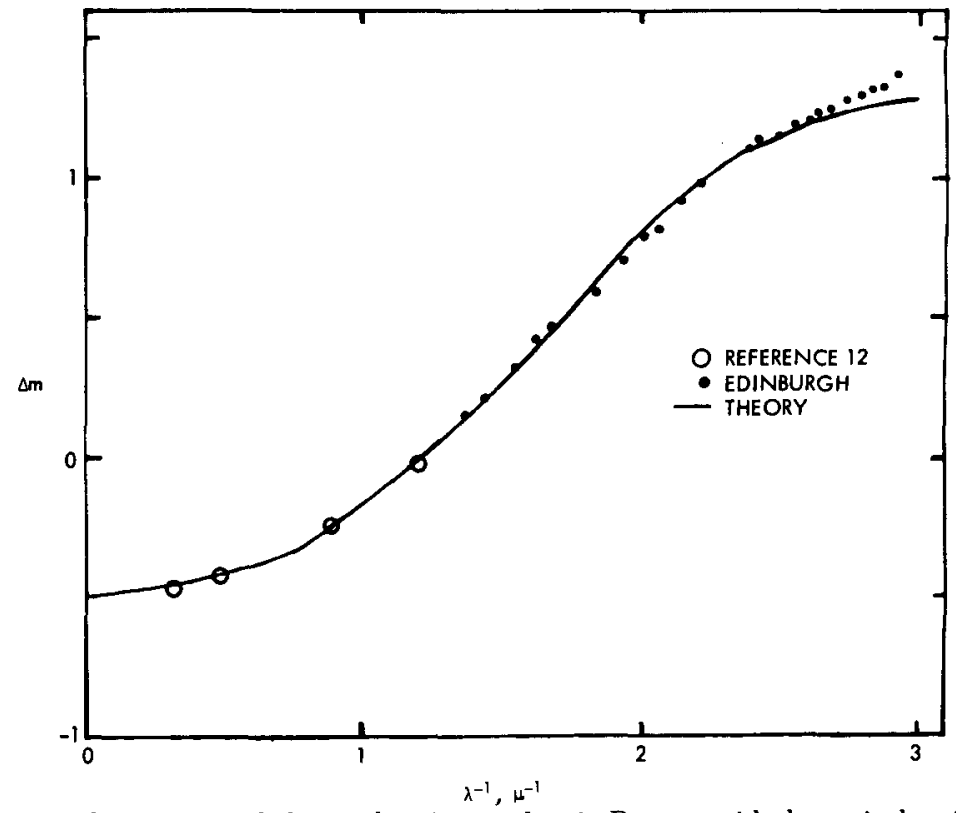

FIGURE 4. - Comparison of observed extinction law in Perseus with theoretical extinction curve. 
in Cygnus and Perseus as obtained in the present investigation and as reported in reference 12 with the theoretical extinction curve. Graphite cores with radii less than 0.06 covered with ice mantles reproduce best the observed Cygnus curve (fig. 3), whereas a Gaussian distribution of pure graphite particles with dispersion of $\pm 0.01 \mu$ centered at radius $a=0.06 \mu$ fits best the Perseus curve (fig. 4). (See ref. 13.) The wavelength $4300 \AA$ is close to that at which the refractive index of graphite particles ceases to be constant. Over the wavelength region $0.8<\lambda^{-1}$ $<2.3$, the extinction of graphite is close to the $\lambda^{-1}$ law and thereafter deviates from the linearity depending on particle sizes. (See ref. 14.) It is difficult to explain the discontinuity in gradient in terms of any known absorption process; the fluctuations in mean particle size in the line of sight may be averaged out to simulate the observed two-straightline effect for the mean reddening curve.

\section{CONCLUSIONS}

In the direction of both Cygnus and Perseus, the extinction is very uniform over a distance of at least $2.5 \mathrm{kpc}$ in the line of sight, but the extinction law in Cygnus is significantly different from that in Perseus. The slope of the ultraviolet part of the curve relative to the blue part decreases in Perseus by about 30 percent as compared with that in the Cygnus region.

The difference between the Cygnus and Perseus laws can be explained if it is assumed that the extinction in Perseus is due to graphite grains and that in Cygnus, to graphite cores with ice mantles. Therefore, a difference in composition may exist between the Cygnus and the Perseus arms.

\section{REFERENCES}

1. Guthrie, B. N. G.; Lawrence, L. C.; Nandy, K.; Pratt, N. M.; and Redoish, V. C.: Spectrophotometry and Multicolor Photometry at the Royal Observatory, Edinburgh. Communication Roy. Obs. Edinburgh, no. 27, 1965.

2. BAKER, E. A.: Spectrophotometric Measurements of Early.Type Stars I. Methods of Observation and Results for Oe 5 Stars. Pub. Roy. Obs. Edinburgh, vol. 1, 1949, p. 15.

3. Nandy, K.: Observations of Interstellar Reddening I. Results for Region in Cygnus. Pub. Roy. Obs. Edinburgh, vol. 3, no. 6, 1964, p. 142.

4. Nandy, K.: Observations of Interstellar Reddening II. Results for Region in Perseus. Pub. Roy. Obs. Edinburgh, vol. 5, no. 2, 1965, p. 13.

5. Rodcers, A. W.: Photoelectric Spectrophotometry of O-Type Stars. Roy. Astron. Soc., Monthly Notices, vol. 122, 1961. p. 413.

6. Whitford, A. E.: The Law of Interstellar Reddening. Astron. J., vol. 63, 1958, p. 201.

7. (;REenberc., J. M.; and Mei.Tzer. A. S.: The Effect of Orientation of Non-Spherical Particles on Interstellar Extinction. Astrophys. J.. vol. 132, 1960, p. 667.

8. Wilson, R.: The Relation Between Interstellar Extinction and Polarization. Roy. Astron. Soc., Monthly Notices, vol. 120, 1960, p. 51.

9. Underhill. A. B.; and WaI.Ker. (;. H.: The Shape of the Interstellar Reddening Curve. Roy. Astron. Soc., Monthly Notices, 1965.

10. Johnson, H. L.: Interstellar Extinction in the Galaxy. Astrophys. J., vol. 141, 1965, p. 923 . 
11. Mendoza. E. E., V: La Ley de Extincion Interestallar en las Pleyades. Tonantazintla y Tacubaya, vol. 4, no. 26. 1965.

12. Johnson. H. L.; and Borgiman. J.: The Law of Interstellar Extinction. Bull. Astron. Inst. Netherlands, vol. 17, 1963, p. 115.

13. NANDY, K.; and Wickramasinghe, N. C.: A Survey of Recent Interstellar Reddening Observations. Pub. Roy. Obs. Edinburgh, vol. 5, no. 3, 1965, p. 29.

14. Wigkramasinghe, N. C.; and Guillaume, C.: Interstellar Extinction by Graphite Grains. Nature, vol. 207, 1965, p. 366.

\section{DISCUSSION}

Field: In your diagram you included the slopes of the reddening law as functions of $\lambda^{-1}$. It makes a difference, doesn't it, how the normalization is carried out there?

Nandy: The shape of the reddening curve does not depend on how it is normalized. The normalization procedure which $I$ have used is as follows: A smooth curve is drawn through the observed points, and the magnitude differences $\Delta m_{\lambda}$ at $\lambda^{-1}=1.34 \mu^{-1}$ and $\lambda^{-1}=2.28 \mu^{-1}$ are read off this curve. The normalizing factor by which all observed magnitude differences are multiplied is the reciprocal of the slope of the line joining the two points $\left(\Delta m_{\lambda}, 1.34 \mu^{-1}\right)$ and $\left(\Delta m_{\lambda}, 2.28 \mu^{-1}\right)$. The zero point of $\Delta m_{\lambda}$ is fixed so that $\Delta m_{\lambda}=1$ at $\lambda^{-1}=2.22 \mu^{-1}$.

O'Dell: Other papers herein will show that there is some physical basis for thinking that the deviations you find might be due to very smallscale changes, primarily the presence of very high luminosity stars. In this case an even better requirement above this (taking all standard stars in the same region) would be to be sure that they are very close in space, because even in Perseus you are looking over a distance of several $\mathrm{kpc}$ and this could be integrating over regions which are generally of a 1-w density and a low mass cutoff region. This is something which I think may account for the differences observed and is something to watch out for.

Strömgren: In that connection, how many stars were measured in the Pleiades?

Nandy: Only one star.

Strömgren: Is this the star that has a high color excess measured by Mendoza?

Nandy: Yes, this is the star HD 23512 (Hertzsprung number 371).

Strömgren: The fact that the reddening is unusually high is of interest in connection with Dr. O'Dell's question.

Wampler: It is listed by Mendoza $\mathrm{V}$ as A0 V. I looked at a spectrum obtained by Helmut Apt and the calcium line appears too strong for A0. If the star is a fairly rapid rotator, some of the other metallic lines may be washed out. They do seem to be present and seem to be rather diffuse. I also looked at Dr. Herbige's plates and came to the same conclusion. Perhaps the spectral type is in fact closer to A2 than A0. 
Nandy: It is spectroscopic binary?

Wampler: I don't know. It is not noted as a spectroscopic binary. Dr. Apt didn't think so at the time. He didn't have it in his list of binaries.

Nandy: This is the difficulty in deriving the reddening curve from one star.

Behr: I have two questions: what are the galactic coordinates of the two fields and what are the diameters or sizes of the fields?

Nandy: The galactic coordinates for Cygnus are $\ell^{11} \approx 70^{\circ}$ and $b^{\mathrm{II}} \approx 1^{\circ}$ and for Perseus, $\ell^{\mathrm{II}} \approx 140^{\circ}$ and $b^{\mathrm{II}} \approx-2^{\circ}$.

Behr: That is exactly in the galactic plane and it is just in the region of high absorption. What is the area?

Nandy: The area is about 30 square degrees in each direction.

Behr: Do you think that these 30 stars are typical for the whole Cygnus arm?

Nandy: No, I do not say that. I am combining my results with Johnson and others. Excluding the Cepheus stars which show anomalous reddening, the majority of stars studied by Johnson and Borgman in the Cygnus arm exhibit the Cygnus reddening curve. The Scorpio-Ophiuchus stars also show Cygnus extinction.

Hall: Some years ago I tried to find out where the polarization in the Perseus direction was produced. There was no evidence of polarization beyond our own spiral arm. If I remember correctly, I tried this also with color excess and came to the conclusion that most of the absorption was in the Orion arm. Maybe I'm wrong on this. Have you tried this? In other words, you may be observing only the effects due to our arm when you observe Perseus.

Nandy: The space distribution of stars in the Perseus direction shows that the highly reddened stars are inside the Perseus arm, whereas the comparison stars are moderately reddened and are near the edge of the Perseus arm. Therefore, the extra extinction of the highly reddened stars, as compared with that of the moderately reddened stars, has probably been produced in the Perseus arm.

Donn: What was the graphite size distribution that you used?

Nandy: For Cygnus, graphite cores with radii less than $0.06 \mu$ covered with ice mantles, whereas for Perseus, the Gaussian distribution of pure graphite particles with a dispersion of $0.01 \mu$ centered at $0.06 \mu$ fairly reproduces the observations.

Wickramasinghe: The precise values are not particularly significant here. It does seem that the Cygnus curve could be reproduced rather insensitively with respect to the core radius, as long as one had an equilibrium distribution of mantles.

Nandy: Yes, I have left this problem for you because you can discuss it much better.

271-992 O-67-3 